\title{
Psicomotricidad, madurez para la lectoescritura, autocontrol y aceptación en preescolares de Huancayo
}

\author{
Psychomotor skills, maturity for literacy, self-control and acceptance in \\ preschool children of Huancayo
}

\begin{abstract}
Luis Alberto Yarlequé Chocas ${ }^{1,2}{ }^{*}$, Eddy Salvador Paredes Flores ${ }^{2}$, Jorge Luis Tapia Camargo ${ }^{2}$, Edith Rocío Nuñez LLacuachaqui ${ }^{2}$, Linda Loren Navarro García ${ }^{2}$, Elvira Janeth Jiménez Asto ${ }^{3}$, Reyna Maricruz Celis Crespo ${ }^{4}$ ' Universidad Continental, ${ }^{2}$ Universidad Nacional del Centro del Perú, ${ }^{3}$ Institución Educativa Estatal $N^{\circ} 577,{ }^{4}$ I. E. Nuestra Señora de las Mercedes
\end{abstract}

\section{RESUMEN}

El objetivo fue establecer si el desarrollo de la psicomotricidad influye en la madurez para la lectoescritura, el autocontrol y la aceptación en preescolares de Huancayo. La investigación es aplicada de nivel teórico. Se desarrolló, experimentó y evaluó los efectos de un programa psicomotor con un diseño cuasi experimental de dos grupos no equivalentes. La población estuvo constituida por estudiantes de 3, 4 y 5 años (II ciclo) de instituciones educativas rural y urbana de Huancayo y la muestra abarcó 179 preescolares de tres instituciones, pero se experimentó en dos, quienes fueron evaluados en las cuatro variables, antes y después del experimento. Los resultados mostraron que los preescolares con mas desarrollo psicomotor alcanzaron mayor madurez para la lectoescritura; ambos grupos mejoraron en su aceptación por los pares así como en su autocontrol. Se concluyo que el programa "estimulando el cerebro y movimiento del niño" favorece el desarrollo de la psicomotricidad; que sí se desarrolla la psicomotricidad, esto influye positivamente en la madurez para la lectoescritura y que no basta la estimulación psicomotriz que vienen recibiendo en las aulas; que los estímulos proporcionados por las actividades que se desarrollan en las aulas, sí permiten que el pequeño aprenda a autorregular su conducta y que en la aceptación de los niños por sus pares, es muy importante el papel que tiene la educación inicial pero que este es potencializado por el programa.

Palabras clave: Psicomotricidad, autocontrol, aceptación, madurez para la lectoescritura.

\begin{abstract}
The objective was to establish whether the development of psychomotor skills influences the maturity for literacy, self-control and acceptance in preschool children of Huancayo. The research is applied on a theoretical level. Its developed, experimented and evaluated the effects of a psychomotor program with a quasi-experimental design of two groups are not equivalent. The population was composed of students of 3, 4 and 5 years (II cycle) of rural and urban educational institutions of Huancayo and the sample comprised 179 preschool children from three institutions, but it was experimented in two, who were evaluated in the four variables before and after the experiment. The results showed that preschoolers with more psychomotor development reached greater maturity for literacy; both groups improved on their acceptance by peers as well as in their self-control. It was concluded that the program "by stimulating the brain and movement of the child" favors the development of psychomotor skills; that the psychomotor skills its develop, this influences positively on the maturity for the literacy and that it is not enough for the psychomotor stimulation that are receiving in the classroom; that the stimuli provided by the activities carried out in the classrooms, it allows that the child learns to regulate his conduct and that in the acceptance of children by their peers, it is very important the role that has the initial education but that this is maximized by the program.
\end{abstract}

Keywords: Psychomotor skills, self-control, acceptance, maturity for literacy.

Historial del artículo:

Recibido, 02 de febrero de 2017; aceptado, 11 de marzo de 2017; disponible en línea, 05 de junio de 2017

* Magíster y Doctor en Psicología, docente en la Universidad Nacional del Centro del Perú y Universidad Continental.

Correo: lych5@yahoo.com 


\section{INTRODUCCIÓN}

El Perú se encuentra en los últimos lugares en las pruebas del Programa para la Evaluación Internacional de Estudiantes (PISA, por sus siglas en inglés), desde fines del siglo pasado (2000-2015). Si bien, el país ha salido del último lugar -puesto 65- alcanzado en el 2012 en comprensión lectora (y en los otros dos rubros evaluados como matemáticas y ciencias), la situación en este rubro continúa deplorable, ocupa el puesto 62 de los 69 países evaluados durante el 2015 (PISA, 2015). Según la Evaluación Censal de Estudiantes (ECE) 2015, reportado por el Ministerio de Educación (2016) a través de la Oficina de la Medición de Calidad de los Aprendizajes, solo el 14,7\% de alumnos del segundo de secundaria, que por primera vez fueron evaluados, lee de manera satisfactoria, en tanto que el $23,7 \%$ de ese cerca de medio millón de estudiantes están en el nivel "previo al inicio"; es decir, carecen de los aprendizajes necesarios para estar en dicho grado. Este nivel de carencia de capacidad de lectura en el referido grado es más dramático en la sierra del país, padecen más de la mitad de escolares en Huancavelica $(52,4 \%)$ y Apurímac (50,4\%), en tanto que en el departamento de Junín el 21,9\% está en esta situación, y en Huancayo, el $15,6 \%$. En la primaria, en la ciudad de Chincha, Maco (2015) encontró niños hasta de sexto grado que no saben leer y que fueron sin embargo promocionados.

Paradójicamente, el Ministerio de Educación ha tomado una serie de medidas que lejos de mejorar el nivel de los educandos, contribuyen a mantener la situación. Además, en el Perú la educación física, es decir la estimulación sistemática de la psicomotricidad, se inicia oficialmente como un logro reciente, en el nivel primario. El gobierno anunció la contratación de 4.500 profesores de Educación Física para 2 mil escuelas (La República, 2015). Si bien esta iniciativa constituye un avance importante en favor de la educación integral del estudiante, no es suficiente, en primer lugar porque ni siquiera abarca toda la educación primaria, y en segundo, porque la educación inicial todavía está al margen de ello, cuando en realidad debería empezar desde ahí. Los investigadores realizaron observaciones en las que pudieron captar que los estudiantes del nivel inicial (II ciclo) muestran dificultades para orientarse en el espacio, controlar su postura, realizar actividades que impliquen coordinación visomanual y podal, además de no seguir el ritmo. También se observó a niños que no se integran a los grupos, hay poca tolerancia a la frustración cuando pierden en algunos juegos y tampoco saben respetar sus reglas.

En la educación básica, y en especial en los niveles inicial y primaria, por mucho tiempo se puso mayor énfasis en otras áreas, aunque sin éxito, y se descuidó erróneamente la educación psicomotriz, pese a que esta considera al individuo en su totalidad, pretende desarrollar al máximo las capacidades individuales valiéndose de la experimentación y ejercitación consciente del propio cuerpo para lograr que el estudiante tenga un mayor conocimiento de las posibilidades en relación consigo mismo y con el medio en que se desenvuelve (Pérez, 2004). Por razones didácticas los teóricos han dividido la psicomotricidad en lo que se ha dado por llamar áreas. Al respecto la mayoría coincide en que tales áreas son: el conocimiento corporal, la lateralidad, la coordinación dinámica, la coordinación estática, la orientación espacial, la coordinación visomotriz y la estructuración espacio temporal. El conocimiento corporal se refiere a la habilidad que permite el reconocimiento de las distintas partes del cuerpo, la nominación y su inclusión en un todo armónico (Yarlequé, 1998).

La lateralidad se refiere al desarrollo y uso preferente de las funciones sensoriales y motrices de los segmentos corporales localizados en un hemicuerpo (Celada y Cairo, 1990).

La coordinación dinámica implica la flexibilidad en el control motor y los mecanismos de ajuste postural que se realizan durante el movimiento (Condemarín, 1984).

La coordinación estática se refiere a la capacidad del niño para mantener su cuerpo en equilibrio durante un cierto tiempo sin movimiento. La orientación espacial supone el reconocimiento y manejo del espacio (Yarlequé, 1998).

La coordinación visomotriz es la capacidad de coordinar la visión con movimientos del cuerpo o sus partes (Frostig, 1980).

La estructuración espacio temporal se refiere a las posibilidades que tiene el niño para producir diversos tipos de ritmos (Yarlequé, 1998; Nieto, 1980).

Por otra parte, el autocontrol ha sido definido por primera vez en 1973 por la American Psychological Association (citado en Fernandes, Marín y Urquijo, 2010) como la habilidad para reprimir o la práctica de represión de reacciones impulsivas de un comportamiento, deseos o emociones. Según Brenner y Salovey (citados en Serrano y García, 2010), es la habilidad para administrar las respuestas que se originan dentro de los componentes cognoscitivos, experimentales, comportamentales, de expresión física y bioquímica en las personas.

El estudiante aceptado es quien recibe el mayor número de valoraciones o nominaciones positivas por parte de sus compañeros. Esta se relaciona con mostrar comportamientos de ayuda, ser considerado por los compañeros, seguir la regla de los juegos e implicarse activamente en la interacción positiva con los iguales, sostienen Trianes, De la Morena y Muñoz (citados en Zavala, Valadez y Vargas, 2008) 
Otra condición que debe lograr el niño en el nivel inicial, para tener éxito en la educación primaria, es la madurez para la lectoescritura, que implica un conjunto de habilidades como discriminación visual y auditiva, relación con un significado, combinación, escritura y pronunciación (Suárez, 1999).

El estudio de estas variables en preescolares es vital porque en las manos de este sector se encontrará el destino del país y sus regiones dentro de $3 \circ 4$ décadas; de ahí la importancia de intervenir sobre el fenómeno. Al respecto se han hecho algunos estudios, en torno a la psicomotricidad se encuentran los trabajos de Zelago y Kolb (citado en Papalia, Wendkos y Duskin, 2010) quienes analizaron el efecto de la estimulación temprana en el desarrollo de la locomoción. Trabajaron con 24 niños durante seis semanas, de la segunda a la octava semana de nacimiento. Constituyeron cuatro grupos: al primero lo sometieron a entrenamiento (se paraba al niño en una superficie plana, favoreciendo sus movimientos podales y sujetándole de los brazos), el segundo grupo recibió entrenamiento pasivo (movimiento de brazos y piernas cuando el niño estaba en la cuna) y el tercero y cuarto, ningún entrenamiento. Hallaron que los niños sometidos a entrenamiento activo aprendían a caminar entre los 10 y 11 meses, mientras que el promedio de los grupos control empezaban a partir de los 12 meses, sin embargo todos aprendieron a caminar en un máximo de 14 meses.

Martinez y Sabater (citados en Pacheco y Romero, 2005) mediante un estudio acerca de problemas escolares encontraron que en los niños de los primeros grados, la inadecuada orientación derecha-izquierda y topográfica le conduce a una confusión de letras y números simétricos tanto en el eje horizontal ( $d, b$ y $p, q)$ como vertical $(v, n$ y 6,9$)$, además los trazos gráficos presentan distorsiones de los ángulos en las figuras geométricas por deficiencia en la estructuración espacio temporal.

Blas, Javier y Yarlequé (1989) llevaron a cabo un estudio acerca del desarrollo psicomotor en niños que vivían en el penal de Santa Mónica. La muestra fue dividida en dos subgrupos (preinicial e inicial). Encontraron diferencias entre ambos grupos. En el grupo de niños más pequeños (preinicial), el área más desarrollada fue la visomanualidad, y en los niños del grupo de inicial se notó un gran desarrollo de las demás áreas con excepción de la coordinación estática.

De la Cruz (1989) realizó una investigación con 68 niños de 5 y 6 años, a los que se les aplicó el test gestáltico visomotor de Bender y el test de madurez para el aprendizaje escolar de Lauster y Lauster. Encontró entre otras cosas, que la madurez para el aprendizaje escolar, está relacionada con la maduración visomotriz y que la variable sexo, no está relacionada con la maduración para el aprendizaje escolar ni con la percepción visomotriz.

Zavala (1991) desarrolló un estudio con 50 niños de 3 a 5 años de zonas urbano marginales, 25 de los cuales adolecían de desnutrición crónica. La muestra fue evaluada con la batería propuesta por Launay, Picq y Vayer. Encontró entre otras cosas, que si bien los niños con déficit nutricional obtenían en la prueba puntajes que tendían a agruparse del promedio hacia abajo, el otro grupo obtenía puntajes que se acumulaban de lo esperado hacia arriba, pero tales diferencias no se registran en todas las áreas, solo en la coordinación visomanual y en el control postural. La autora hizo notar que en ambos grupos se registraron déficit en organización perceptiva y conocimiento del cuerpo y que ambos obtuvieron puntajes elevados en coordinación dinámica general, lo cual fue explicado por las condiciones de vida (geográficas y económicas de los niños).

Escobar (citado en Pacheco y Romero, 2005) en su estudio acerca de los juegos motores en niños de 5 años, halló que los juegos motores influyen positivamente en el desarrollo de la psicomotricidad.

Javier (1994), desarrollo un estudio comparativo acerca de la psicomotricidad de niños en edad preescolar de las ciudades de Huancayo y bellavista. La autora encontró entre otras cosas que en la coordinación visomanual, los niños de tres años de ambas ciudades difieren entres sí, en relación al conocimiento corporal existen diferencias a favor de Huancayo, pero al ser evaluados en reconocimiento de sus partes, no existen diferencias en esta área, Finalmente en la lateralidad no existen diferencias.

Canaviri y Casaño (citadas en Alanya y Núñez, 2006), desarrollaron una investigación acerca de los efectos del programa de psicomotricidad en el desarrollo de la coordinación visomanual. Ellas reportaron que el programa mejoró el desarrollo de la coordinación visomanual.

Pacheco y Romero (2005) experimentaron un programa para la estimulación psicomotriz en niños de 3 años, hallándose diferencias significativas en el grupo experimental, es decir el programa propuesto ayudó a la maduración de las áreas de la psicomotricidad.

Cotom (2012) investigó sobre la psicomotricidad y su relación en el proceso de lectoescritura, el estudio utilizó la prueba $A B C$ para medir el nivel de madurez del aprendizaje por medio de diferentes áreas de la psicomotricidad. La autora llega a la conclusión de que la aplicación de ejercicios y actividades con secuencia y constancia para el aprendizaje estimulan, desarrollan y favorecen al rendimiento del niño en cada una de las facetas de su vida, desde lo emocional, 
intelectual, familiar, económico hasta social, formando una persona integral. Por lo tanto, la estimulación de las áreas de psicomotricidad mejora significativamente el comportamiento y las relaciones interpersonales del niño.

Salazar (2013) realizó un estudio de carácter descriptivo sobre la psicomotricidad y su incidencia en la lectoescritura de los niños del primer año de educación básica del jardín de infantes "Club Árabe Ecuatoriano" de la ciudad de Quito. Las técnicas que utilizó la autora fueron la prueba de habilidades de las áreas de la psicomotricidad y la prueba de niveles de maduración de la lectoescritura. En el estudio se concluyó que es necesario adquirir todas las áreas de la psicomotricidad para tener bases en el aprendizaje de la lectoescritura.

Respecto de la madurez para la lectoescritura, se tienen los estudios de Guevara (1980), quien experimentó en dos grupos de niños de cinco años con inmadurez para el aprendizaje de la lectoescritura. Luego de seis meses de trabajo, al aplicar un postest que en el grupo de experimento que en el pretest había alcanzado una media equivalente a un nivel inferior de madurez; esta medida en el postest se incrementó casi el $100 \%$, mientras en el grupo control el incremento fue de 36 $\%$. Además, se observó en los niños del experimento que los tiempos de trabajo habían aumentado notablemente, siendo este hecho un signo más de madurez para el aprendizaje de la lectoescritura.

Soto (1986) realizó un estudio con 120 niños en edad preescolar, 60 de estatus socioeconómico alto, de ambos sexos, a los que se aplicó el test de figuras geométricas de Gesell y el ABC de Filho (1925). Encontró que el estatus socioeconómico no tiene un papel distintivo en la percepción visomotriz, pero sí se hallan diferencias entre ambos grupos, en la madurez para el aprendizaje de la lectoescritura. Comprobó además que los varones presentan un mayor nivel de madurez para el aprendizaje de la lectoescritura. Por último, los niños de 6 años obtuvieron resultados superiores significativamente en ambas pruebas que el que alcanzaron los niños de 5 años.

Gonzáles y Romero (1999) al analizar la influencia del adecuado e inadecuado desarrollo de la producción fonológica y la articulación de los fonemas castellanos en relación a la variable aprendizaje lector, encontraron que la producción fonológica es una variable predictiva de la lectura entre los 6 y 8 años, siendo la exactitud lectora la variable que mejor explica la producción fonológica entre estas edades.

Piacente, Marder, Resches y Ledesma (2005) en su investigación sobre los conocimientos y habilidades prelectoras de niños provenientes de familias beneficiarias y no beneficiarias de planes sociales y de las relaciones que guardan con el nivel intelectual y con los contextos de crianza. Constataron entre otras cosas que hay asociaciones positivas entre las habilidades prelectores y los contextos de crianza en ambos grupos. En cuanto a las relaciones entre las habilidades y conocimientos infantiles y el nivel intelectual, el comportamiento de ambos grupos fue diferente. El peso relativo del desempeño cognitivo sobre los resultados obtenidos en alfabetización ha mostrado tener importancia relevante para el grupo de beneficiarios y ser de escasa incidencia para el grupo de no beneficiarios.

En España, González (citada en Alvino, 2010) desarrolló una investigación sobre la comprensión lectora en niños: morfosintaxis y prosodia en acción. La autora demostró que el entrenamiento en prosodia manifiesta su eficacia en la mejora de la comprensión lectora ya que sirve como prerrequisito en las habilidades de decodificación fluida de palabras o la fluidez de la lectura de textos en los niños con un nivel lector bajo, logrando un incremento en la velocidad de acceso a los códigos fonológicos y la mejora de la expresividad. Utilizó el test breve de inteligencia de Kaufman, prueba de evaluación de retraso en lectura y test de eficiencia lectora que fue aplicado a 67 estudiantes con el método experimental y el diseño preprueba y posprueba con grupo control.

Morales (citado en Maco, 2015) investigó acerca de los factores socioculturales que predicen la adquisición de la alfabetización lectora de los niños de Lima. Trabajó con una muestra de 314 alumnos de niveles socioeconómicos medio y bajo, evaluando la decodificación, vocabulario, motivación hacia la lectura, estrategias de lectura, madurez intelectual, género, clima alfabético del hogar y nivel socioeconómico. Los resultados muestran que el vocabulario y la decodificación se correlacionan significativamente con la alfabetización lectora, mostrándose como factores importantes para el desarrollo de la comprensión de lectura. El nivel socioeconómico tiene un efecto directo y significativo en la comprensión de lectura y que es un predictor del nivel de vocabulario.

Pugh (2014) reporta un experimento con 14 lectores simultáneos que aprendieron a leer dos idiomas a los 5 años y 10 lectores secuenciales que aprendieron hindi a los 5 años y a los 10 años el inglés. Encontró que la diferencia de idiomas en los que se lee no generan diferencias en las zonas dorsales y ventrales; ambas se activan más o menos del mismo modo. Pero la lectura en inglés activa preferentemente el giro temporal inferior izquierdo, mientras que el hindi activa preferentemente el lóbulo parietal inferior izquierdo. Los lectores secuenciales mostraron mayor activación del lóbulo parietal inferior izquierdo. El autor sugiere que la exposición temprana simultánea a la lectura de distintas ortografías da como resultado mayor plasticidad neural. 
En relación al autocontrol se tienen los trabajos de Gómez y Luciano (1991), quienes realizaron un estudio cuyo propósito fue comparar la adquisición, mantenimiento y generalización de una conducta de autocontrol en niños pequeños. Los resultados mostraron la efectividad de ambos procedimientos ("aumento gradual de demora" y "decir-hacer"); el procedimiento "decir-hacer" ha mostrado generalización funcional a través de diferentes tareas, y además este último sistema ha creado conductas alternativas en los tiempos de espera. El mantenimiento de la conducta de espera, al eliminar el agente que señalaba el final de la espera, se ha producido en dos de los sujetos, reduciéndose el tiempo de espera en el tercero paulatinamente, aunque el efecto del mantenimiento de "demora gradual" no se ha podido aislar por la generalización mencionada del procedimiento "decir-hacer"; no obstante los datos recogidos de mantenimiento han sido escasos. La aplicación del procedimiento "decir-hacer" como un repertorio introducido en una conducta de autocontrol es novedoso, siendo entendido como una ampliación reconceptualizada de los sistemas de autoinstrucciones clásicas.

Herrera y Tejidor (2004) en Venezuela estudiaron el desarrollo del autocontrol en niños preescolares con comportamiento asociado al TDA/H, a quienes se les somete a un programa pedagógico centrado en el autocontrol. En la investigación trabajaron como muestra a un solo niño de 4 años y 4 meses; los resultados muestran un progreso en algunas conductas de autocontrol, pasando de "nunca" a "a veces"; $\sin$ embargo, las conductas se vuelven a manifestar en presencia de la docente regular, por lo que concluyen que el comportamiento responde a una necesidad imperiosa de llamar la atención de quienes lo rodean. Además destacan la posibilidad de integrar a un niño con rasgos asociados al TDA/H aplicando estrategias pedagógicas por un tiempo prolongado y de forma permanente.

También Zavala et al. (2008) estudiaron la inteligencia emocional y las habilidades sociales en adolescentes con alta aceptación social, encontrando diferencias significativas a favor del grupo con alta aceptación social en la inteligencia emocional. En este mismo grupo se hallaron puntuaciones bajas en habilidades sociales, siendo más baja la habilidad para hacer peticiones.

Fernandes et al. (2010) investigaron las relaciones entre el autocontrol y el autoconcepto en 1213 niños, de ambos sexos, de segundo a octavo grado de una escuela pública, con edades variando de 8 a 14 años. Han sido observadas correlaciones positivas y significativas entre los autoconceptos personal y social y todas las medidas. En relación al autoconcepto escolar hubo asociación negativa con el autocontrol en los varones, mientras que en las niñas la relación se ha dado apenas con el autocontrol de los sentimientos y emociones. Además, han sido evidenciadas correlaciones negativas y significativas entre el autoconcepto familiar y el autocontrol que se refiere a las normas y conductas sociales y de forma general en los varones. En las niñas, esa asociación se ha observado con el autocontrol que se refiere a las normas y conductas sociales, como también con el autocontrol general.

Serrano y García (2010) realizaron un estudio en el que se pudo establecer niveles de presencia de la competencia emocional Autocontrol correspondiente a la variable Inteligencia Emocional en adolescentes de ambos sexos. Se aplicó un estudio descriptivo con diseño de campo y transeccional contemporáneo, sobre la población de estudiantes en edades comprendidas entre los 16 y 18 años, cursando el último grado de secundaria de instituciones educativas privadas mixtas, basado en los principios acerca de la inteligencia emocional y las teorías del desarrollo que explican la adolescencia. Se concluyó que no existen diferencias entre los estudiantes adolescentes de ambos sexos en cuanto a la competencia emocional Autocontrol.

Arana (2014) presenta un estudio del autocontrol y su relación con la autoestima en adolescentes del segundo grado básico del instituto Dr. Werner Ovalle López de Quetzaltenango, en el que demuestra que no existe una relación significativa entre ambas variables, lo que indica que una no es consecuencia de la otra; asimismo presenta una propuesta para mejorar el nivel de autocontrol y autoestima en los adolescentes evaluados.

Pese a todos los trabajos realizados, en ninguno de ellos se ha relacionado las cuatro variables que aquí se estudian. El objetivo general fue establecer si influye el desarrollo de la psicomotricidad en la madurez para la lectoescritura, el autocontrol y la aceptación en preescolares de Huancayo.

Los objetivos específicos fueron a) proponer un programa para el desarrollo psicomotor en preescolares de Huancayo, b) experimentar el programa psicomotor "estimulando el cerebro y movimiento del niño" en preescolares de Huancayo, c) evaluar los efectos del programa "psicomotor" en la madurez para la lectoescritura, autocontrol y aceptación en preescolares de Huancayo.

La hipótesis general sostuvo que, el desarrollo de la psicomotricidad sí influye en la madurez para la lectoescritura, autocontrol y aceptación en preescolares de Huancayo. Las hipótesis específicas: a) el programa "estimulando el cerebroy movimiento del niño" favorece el desarrollo de la psicomotricidad en preescolares de Huancayo; b) el desarrollo de la psicomotricidad influye en la madurez para la lectoescritura en preescolares de Huancayo; c) el desarrollo de la psicomotricidad influye en el autocontrol en preescolares de Huancayo; 
d) el desarrollo de la psicomotricidad influye en la aceptación en preescolares de Huancayo.

\section{MATERIAL Y MÉTODOS}

La investigación es aplicada y de nivel explicativo o teórico (Sánchez y Reyes, 2006). El método empleado fue el experimental con diseño cuasi experimental, de dos grupos no equivalentes. (Yarlequé y Cerrón, 2011)

El esquema es el siguiente:

\begin{tabular}{lll} 
Grupo experimental & Olabc $\times$ & O2abc \\
\cline { 2 - 3 } Grupo control & O3abc & O4abc
\end{tabular}

Donde:

O1 Es observación de la muestra en las variables psicomotricidad, madurez para la lectoescritura, autocontrol y aceptación en preescolares de Huancayo antes del experimento.

O2 Es observación de las referidas variables después del experimento.

O3 Es observación de las variables mencionadas del grupo control.

O4 Es observación del grupo control en las mismas variables.

Los subíndices a, b, c cada $\bigcirc$ nos indican las observaciones obtenidas en cada una de las variables.

$X$ es el programa "estimulando el movimiento $y$ cerebro del niño".

La población estuvo constituida por estudiantes de 3,4 y 5 años (II ciclo) de instituciones educativas rural y urbana de Huancayo. La muestra fue de 179 estudiantes de 3 instituciones educativas estatales del nivel inicial en las edades señaladas. Se experimentó en dos de ellas: Institución Educativa $N^{\circ}$ 384 de Sapallanga (rural) y la Institución Educativa Cooperativa Santa Isabel de San Carlos (urbana). La muestra fue seleccionada de modo no probabilístico y por accesibilidad, ya que se requería el consentimiento informado de las docentes de aula (Kazdin, 2001).

La variable independiente fue el desarrollo psicomotor, las dependientes fueron la madurez para la lectoescritura, el autocontrol y la aceptación. Como en el experimento había que probar si el desarrollo psicomotor favorecía el desarrollo de las otras variables, entonces se tenía dos opciones: evaluar a los niños, esperar que ocurra el desarrollo psicomotor y una vez comprobado ello, establecer si tal desarrollo favorecía el de las variables dependientes; pero como eso hubiera requerido mucho tiempo en espera, se optó por elaborar un programa que pudiese lograr el desarrollo psicomotor en breve plazo, con lo que se evitaría dicha espera. Así se desarrolló y experimentó el programa "estimulando el cerebro y movimiento del niño" que a su vez actuó como variable independiente respecto del desarrollo psicomotor.

El programa consta de 25 sesiones que abarcan 7 áreas (el conocimiento corporal, la lateralidad, la coordinación dinámica, la coordinación estática, la orientación espacial, la coordinación visomotriz y la estructuración espacio temporal). Las variables de control fueron la edad cronológica (3-5 años), el nivel educativo (inicial), la modalidad educativa (educación básica regular) y el tipo de institución educativa (estatal).

Las técnicas empleadas fueron la observación indirecta no reactiva y la técnica psicométrica. Y para evaluar la aceptación, se empleó la técnica del sociograma, que consistió en pedir a los niños que nombren a los compañeros con los que prefieren jugar, así se estableció quienes eran los niños más aceptados y menos aceptados.

Para evaluar la psicomotricidad se utilizó una lista de cotejo desarrollada por los investigadores tomando como base el test de Oseretzky La lista de cotejo tiene validez de contenido.

Para evaluar el autocontrol se utilizó la observación directa e indirecta, poniendo al niño en la necesidad de controlar el impulso de comerse un chocolate para obtener otro. El equipo empleado fue una cámara de video y el registro anecdótico del observador.

Para evaluar madurez para la lectoescritura, se empleó el test ABC de Filho (1925). La validez se estableció por el sistema ítem test con coeficiente de 0,59. La confiabilidad fue establecida por Arias y Caycho (2013) con el sistema alfa de Cronbach con coeficiente de 0,677 .

Las técnicas de procesamiento de datos empleadas fueron las medidas de tendencia central, como la media aritmética, y de dispersión, la desviación estándar. La contrastación de hipótesis fue realizada mediante la prueba † de Student, para la comparación de medias y la ji al cuadrado para la comparación de frecuencias.

\section{RESULTADOS}

$H 1$. Existen diferencias significativas entre las medias de las evaluaciones de entrada y salida en la prueba de psicomotricidad del grupo control (tabla 1). 
Tabla 1

Comparación de medias en el pre y postest de la variable psicomotricidad del grupo control.

\begin{tabular}{ccccccccr}
\hline $\mathrm{X} 1$ & $\mathrm{X} 2$ & $\mathrm{DS1}$ & $\mathrm{DS} 2$ & $\mathrm{NC}$ & $\mathrm{GL}$ & $\mathrm{T \dagger}$ & $\mathrm{Tp}$ & Diagnóstico \\
\hline 56,826 & 64,283 & 13,471 & 10,498 & 0,05 & 45 & 2,021 & 0,003 & No significativo \\
\hline
\end{tabular}

Tabla 2

Comparación de medias en el pre y postest de la variable psicomotricidad del grupo experimental.

\begin{tabular}{ccccccccc}
\hline $\mathrm{X} 1$ & $\mathrm{X} 2$ & $\mathrm{DS} 1$ & $\mathrm{DS} 2$ & $\mathrm{NC}$ & $\mathrm{GL}$ & $\mathrm{Tt}$ & $\mathrm{Tp}$ & Diagnóstico \\
\hline 49,459 & 69,692 & 11,039 & 8,056 & 0,05 & 132 & 1,98 & 2,1 & significativo \\
\hline
\end{tabular}

Tabla 3

Comparación de medias por edad en la evaluación pre de la variable psicomotricidad-grupo experimental.

\begin{tabular}{rccccccccccc}
\hline Edades & N1 & N2 & X1 & X2 & DS1 & DS2 & NC & GL & Tt & Tp & Diagnóstico \\
\hline 3 con 4 & 33 & 44 & 36,636 & 57,341 & 10,848 & 7,126 & 0,05 & 75 & 2,000 & 5,3 & Significativo \\
4 con 5 & 44 & 56 & 57,341 & 50,821 & 7,126 & 5,834 & 0,05 & 98 & 2,000 & 4,5 & Significativo \\
3 con 5 & 33 & 56 & 36,636 & 50,821 & 10,848 & 5,834 & 0,05 & 87 & 2,000 & 1,5 & No significativo \\
\hline
\end{tabular}

Tabla 4

Comparación de medias por edad en la evaluación post de la variable psicomotricidad-grupo experimental.

\begin{tabular}{cccccccccccc}
\hline Edades & N1 & N2 & X1 & X2 & DS1 & DS2 & NC & GL & Tt & Tp & Diagnóstico \\
\hline 3 con 4 & 33 & 44 & 63.939 & 69,727 & 7,119 & 9,322 & 0,05 & 75 & 2,000 & 0,003 & No significativo \\
4 con 5 & 44 & 56 & 69.727 & 73,054 & 9,322 & 5,192 & 0,05 & 98 & 2,000 & 0,037 & No significativo \\
3 con 5 & 33 & 56 & 63.939 & 73,054 & 7,119 & 5,192 & 0,05 & 87 & 2,000 & 4,100 & Significativo \\
\hline
\end{tabular}

Tabla 5

Comparación de medias en el pre y postest de la variable madurez para la lectoescritura del grupo control.

\begin{tabular}{ccccccccc}
\hline $\mathrm{X} 1$ & $\mathrm{X} 2$ & $\mathrm{DS} 1$ & $\mathrm{DS} 2$ & $\mathrm{NC}$ & $\mathrm{GL}$ & $\mathrm{Tt}$ & $\mathrm{Tp}$ & Diagnóstico \\
\hline 5,239 & 7,37 & 2,478 & 3,611 & 0,05 & 45 & 2,021 & 0,001 & No significativo \\
\hline
\end{tabular}

Leyenda:

$\mathrm{X} 1$ : media uno

$\mathrm{X} 2$ : media dos.

DS1 : desviación estándar uno.

DS2 : desviación estándar dos.

NC : nivel de confianza.

Gl : grados de libertad

Tt : t teórica

Tp : † práctica

H2. Existen diferencias significativas entre las medias de las evaluaciones pre y post experimentales en la prueba de psicomotricidad del grupo experimental (tabla 2).

H3. Existen diferencias significativas entre las medias de las evaluaciones preexperimentales en la prueba de psicomotricidad del grupo experimental según edad (tabla 3).

H4. Existen diferencias significativas entre las medias de las evaluaciones posexperimentales en la prueba de psicomotricidad del grupo experimental según edad (tabla 4).

H5. Existen diferencias significativas entre las medias de las evaluaciones de entrada y salida en test de Filho del grupo control (tabla 5).

H6. Existen diferencias significativas entre las medias de las evaluaciones pre y posexperimentales en el test de Filho del grupo experimental (tabla 6).

H7. Existen diferencias significativas entre las medias de las evaluaciones preexperimentales en el test de Fhilo del grupo experimental según edad tTabla 7). 
Tabla 6

Comparación de medias en el pre y postest de la variable madurez para la lectoescritura del grupo experimental.

\begin{tabular}{ccccccccc}
\hline $\mathrm{X} 1$ & $\mathrm{X} 2$ & $\mathrm{DS1}$ & $\mathrm{DS} 2$ & $\mathrm{NC}$ & $\mathrm{GL}$ & $\mathrm{Tt}$ & $\mathrm{Tp}$ & Diagnóstico \\
\hline 4,549 & 7,015 & 3,001 & 3,3325 & 0,05 & 132 & 1,98 & 9,94 & Significativo \\
\hline
\end{tabular}

Tabla 7

Comparación de medias por edad en la evaluación pre de la variable madurez para la lectoescritura-grupo experimental.

\begin{tabular}{cccccccccccc}
\hline Edades & N1 & N2 & X1 & X2 & DS1 & DS2 & NC & GL & Tt & Tp & Diagnóstico \\
\hline 3 con 4 & 33 & 44 & 1,879 & 3,82 & 2,16 & 2,170 & 0,05 & 75 & 2,000 & 0,000 & No significativo \\
4 con 5 & 44 & 56 & 3,818 & 6,70 & 2,17 & 2,411 & 0,05 & 98 & 2,000 & 1,037 & No significativo \\
3 con 5 & 33 & 56 & 1,879 & 6,70 & 2,16 & 2,411 & 0,05 & 87 & 2,000 & 8,002 & Significativo \\
\hline
\end{tabular}

Tabla 8

Comparación de medias por edad en la evaluación post de la variable madurez para la lectoescritura-grupo experimental.

\begin{tabular}{cccccccccccc}
\hline Edades & N1 & N2 & X1 & X2 & DS1 & DS2 & NC & GL & Tt & Tp & Diagnóstico \\
\hline 3 con 4 & 33 & 44 & 3,606 & 6,27 & 1,95 & 2,266 & 0,05 & 75 & 2,000 & 4,556 & Significativo \\
4 con 5 & 44 & 56 & 6,273 & 9,61 & 2,27 & 2,484 & 0,05 & 98 & 2,000 & 3,484 & Significativo \\
3 con 5 & 33 & 56 & 3,606 & 9,61 & 1,95 & 2,484 & 0,05 & 87 & 2,000 & 9,686 & Significativo \\
\hline
\end{tabular}

Tabla 9

Comparación de medias en el pre y postest de la variable autocontrol del grupo control.

\begin{tabular}{ccccccccc}
\hline $\mathrm{X} 1$ & $\mathrm{X} 2$ & $\mathrm{DS} 1$ & $\mathrm{DS} 2$ & $\mathrm{NC}$ & $\mathrm{GL}$ & $\mathrm{Tt}$ & $\mathrm{Tp}$ & Diagnóstico \\
\hline 0,4348 & 2,457 & 0,958 & 1,13 & 0,05 & 45 & 2,021 & 6,24 & Significativo \\
\hline
\end{tabular}

Tabla 10

Comparación de medias en el pre y postest de la variable autocontrol del grupo experimental.

\begin{tabular}{ccccccccc}
\hline $\mathrm{X} 1$ & $\mathrm{X} 2$ & $\mathrm{DS} 1$ & $\mathrm{DS} 2$ & $\mathrm{NC}$ & $\mathrm{GL}$ & $\mathrm{Tt}$ & $\mathrm{Tp}$ & Diagnóstico \\
\hline 1,188 & 1,85 & 1,366 & 1,417 & 0,05 & 132 & 1,98 & 6,68 & Significativo \\
\hline
\end{tabular}

Tabla 11

Comparación de frecuencias del pre y postest del grupo control en la variable aceptación por los pares

\begin{tabular}{cccccc}
\hline$N$ & $N C$ & $G L$ & Chi teórica & Chi práctica & Diagnóstico \\
\hline 46 & 0,05 & 9 & 16,919 & 46,110 & Significativo \\
\hline
\end{tabular}

Tabla 12

Comparación de frecuencias del pre y postest del grupo experimental en la variable aceptación por los pares

\begin{tabular}{cccccc}
\hline$N$ & $N C$ & $G L$ & Chi teórica & Chi práctica & Diagnóstico \\
\hline 133 & 0,05 & 12 & 21,026 & 169,081 & Significativo \\
\hline
\end{tabular}

H8. Existen diferencias significativas entre las medias de las evaluaciones posexperimentales en el test de Fhilo del grupo experimental según edad (tabla 8).

H9. Existen diferencias significativas a favor del postest entre las medias del grupo control en la prueba de autocontrol (tabla 9).

H10. Existen diferencias significativas a favor del postest entre las medias del grupo experimental en la prueba de autocontrol (tabla 10). 
H11. Existen diferencias significativas entre las frecuencias del grupo control en el pre y postest en aceptación por los pares (tabla 11).

H12. Existen diferencias significativas entre las frecuencias del grupo experimental en el pre y postest en la aceptación por los pares (tabla 12).

\section{DISCUSIÓN}

Los resultados expuestos han conducido a establecer que no existen diferencias estadísticas entre las evaluaciones pre y posexperimentales del grupo control en psicomotricidad (tabla 1) pero sí las hay en el grupo experimental (tabla 2). Ello significa que el programa "estimulando el cerebro y movimiento del niño" sí tuvo un efecto positivo sobre el desarrollo psicomotor de los estudiantes tal como se había previsto. Pero más aún, en el grupo experimental, antes del experimento no había diferencias entre los grupos de tres y cinco años (tabla 3), pero luego sí las hubo (tabla 4).

Este hecho es muy importante, pues la mayoría de los estudiantes que estaban en el aula de cinco, iban a cumplir seis años a lo largo del año o ya los habían cumplido y lo que es peor, el siguiente año serían promovidos al nivel primario aun cuando psicomotrizmente no se diferenciaran de niños que aún tenían tres o cuatro. De modo que el experimento permitió que los niños que culminaban la educación inicial alcanzaran en conjunto un desarrollo psicomotor significativamente superior al que presentaban los niños de 3, pese a que estos también registraron incremento en sus puntajes. El caso muestra entre otras cosas que la edad cronológica por sí sola no necesariamente marca diferencias entre unos y otros subgrupos, es más, hay casos en que un grupo de menor edad puede presentar mayor desarrollo psicomotor que un grupo de más edad, como pudo notarse en la evaluación preexperimental entre los niños de cuatro y cinco años en la que los de cuatro tenían una media estadísticamente superior que la de los de cinco (tabla 3). El experimento permitió corregir este fenómeno.

Ahora bien, logrado este primer paso, según lo propuesto se procedió a comparar la madurez para la lectoescritura antes y después del experimento en ambos grupos. Tampoco se halló diferencias entre las evaluaciones pre y posexperimentales del grupo control en la prueba de madurez para la lectoescritura, evaluada a través del test de Filho (tabla 5). En el grupo experimental, en cambio, sí hubo diferencias después de la intervención (tabla 6), lo cual confirmaría la idea de que al desarrollarse la psicomotricidad se favorece también la madurez para la lectoescritura. Pero, además, es notable que en este grupo, antes del experimento solo se registró diferencias entre los subgrupos de tres y cinco años (tabla 7). Después del experimento las hubo entre los tres grupos etarios, lo que significa que el programa tuvo efectos positivos sobre los tres subgrupos (tabla 8) y reguló la relación entre ellos, ya que al término del experimento, el grupo de cinco ostentaba diferencias significativas sobre los grupos de cuatro y tres. Pero además el grupo de cuatro se mostró superior al de tres. Este resultado mostraría entre otras cosas que las diferencias en madurez para la lectoescritura no necesariamente están definidas por la edad cronológica sino más bien por la estimulación que recibe el niño.

En lo referente al autocontrol, en ambos grupos se halló diferencias estadísticas entre las evaluaciones pre y posexperimentales y lo mismo ocurre en la aceptación.

Ahora es conveniente analizar cada uno de estos resultados.

El hecho de que no hayan diferencias estadísticas entre los puntajes que obtienen los estudiantes del grupo control en las evaluaciones pre y posexperimentales de la psicomotricidad (tabla 9) y sí las haya en los estudiantes del grupo experimental (tabla 10), probaría que el programa aplicado (estimulando el cerebro y movimiento del niño) sí favorece el desarrollo de la psicomotricidad y no solo eso, sino que pondría de manifiesto también que si bien es cierto que, en los momentos del trabajo en aula en el II ciclo se considera a la psicomotricidad, esto no está siendo lo suficientemente efectivo, ya que los niños al culminar el año escolar no difieren significativamente respecto de cómo ingresaron en lo que a la psicomotricidad se refiere y ratifica nuestra idea de que es necesario trabajar sistemáticamente con la psicomotricidad a fin de lograr resultados que marquen la diferencia.

Resultados análogos respecto de los efectos de los programas sobre la psicomotricidad han sido reportados por Escobar (citado en Pacheco y Romero, 2005); Canaviri y Casaño (citadas en Alanya y Nuñez, 2006); Pacheco y Romero (2005); Launay y colaboradores (citados en Javier, 1988); Zelago y Kolb (citados en Papalia et al. 2010); Maya (citado en Oramas, 2000); Freitez, López y Stornaivolo (citados en Oramas, 2000); Gastiaburú (2012), entre otros. De modo que, en sentido estricto, nuestros hallazgos a este respecto eran en cierto modo esperados. Más aún si se tiene en cuenta que en el experimento las sesiones del programa fueron desarrolladas por estudiantes de la Escuela Profesional de Educación Física y Psicomotricidad, de la Facultad de Educación de la Universidad Nacional del Centro del Perú, asesorados por dos docentes de esa escuela, por un psicólogo investigador del área, dos docentes del nivel inicial, además de una comunicadora. Esto probaría, que hay necesidad de un trabajo multidisciplinario 
para la estimulación adecuada del área psicomotriz y que no basta con que la docente de aula haga ciertas actividades cotidianas sin una consistente planificación. De ello se desprende que la educación física en el Perú no debería comenzar en primaria, sino por lo menos desde el nivel inicial. Afortunadamente, esta idea ya ha sido captada por el Ministerio de Educación y en la propuesta del Diseño Curricular Nacional del 2017 se plasma.

En nuestro trabajo, el programa estuvo constituido por 25 sesiones que se desarrollaban a razón de dos veces por semana. Este programa abarcaba: el conocimiento corporal, la lateralidad, la coordinación dinámica, la coordinación estática, la orientación espacial, la coordinación visomotriz y la estructuración espacio temporal. Cabe destacar que si los experimentadores lo creían pertinente, una sesión se podía repetir con la finalidad de que los participantes logren el objetivo de la misma. Probablemente esto último jugó un papel muy importante en los resultados expuestos y constituiría un elemento sobre el que los docentes deberían reflexionar, pues muchas veces por cumplir el calendario, desarrollan la sesión y pasan a la siguiente, aun cuando no se hayan logrado las metas. Desde la perspectiva con la que aquí se ha trabajado, la sesión en sí misma no tiene sentido si no es porque a través de ella se logra las metas establecidas. Todo esto por supuesto se distancia mucho de algunas actividades motoras que suele realizar la maestra en las aulas del nivel inicial y por consiguiente es perfectamente explicable las diferencias apuntadas más arriba como producto del experimento.

Veamos ahora los aspectos más particulares de los resultados aquí expuestos.

El no haber encontrado diferencias en la madurez para la lectoescritura entre las evaluaciones pre y posexperimental del grupo control y sí haberlas encontrado en el grupo experimental, pondría de manifiesto que el programa en cuestión no solo influye positivamente en la psicomotricidad sino que también, como lo habíamos supuesto al desarrollarse la psicomotricidad, favorece la madurez para la lectoescritura. Resultados análogos fueron reportados por Guevara (1980) y Salazar (2013). De esto se desprenden dos cuestiones: 1) que los niños del II ciclo requieren estimulación psicomotriz para alcanzar la madurez necesaria para el aprendizaje de la lectoescritura, y 2) que sin esta estimulación, aunque tenga la edad requerida, pueden no alcanzarla, como lo demostró Gómez (citado en Chávez y Ramos, 2014). Esto tiene enormes implicancias, pues de ser así, se hace insoslayable la necesidad de iniciar la educación del movimiento (educación física) en el nivel inicial.

Por otro lado explicaría por qué niños que tienen 6 años cumplidos e ingresan a la primaria, con el único requisito de la edad cronológica, tienen tantas dificultades para el aprendizaje de la lectoescritura como lo comprobaron Sánchez y Reyes (2015) y Maco (2015), y también por qué niños con 5 años, que han tenido una adecuada estimulación, pueden leer y escribir sin mayores dificultades (Núñez, 2015). Este mismo fenómeno, como se ha dicho, ha sido reportado en Guadalajara por Gómez (citado en Chávez y Ramos, 2014). También en ese país no se da educación física en el nivel inicial.

Nuestros resultados deberían constituir por lo menos un motivo de reflexión para los representantes del Ministerio de Educación peruano, que al respecto vienen tomando una serie de medidas en torno al aprendizaje de la lectoescritura, así como el ingreso a la primaria a través de la Resolución Ministerial 5562014 (MINEDU, 2014): limitar el aprendizaje de la lectoescritura en preescolares y prohibir el ingreso de los niños que no tienen 6 años cumplidos al 31 de marzo sin tomar en cuenta su desarrollo psicomotriz, emocional, social ni cognitivo, basadas en lo que Núñez (2015) ha denominado cronologismo. En el Perú esta situación intentos ser mejorada por el Congreso que mediante ley propuso ampliar el plazo hasta el 31 de julio, pero tal ley fue observada por el Poder Ejecutivo a instancias del Ministerio de Educación. Nuestros hallazgos contradicen el cronologismo que viene aplicando el Ministerio de Educación y coinciden con lo propuesto por Fihlo, porque en efecto no se trata de la edad cronológica sino más bien de evaluar a cada niño y establecer si tiene o no los requisitos que le dan posibilidades de enfrentar con éxito el primer grado, tal como lo hemos propuesto. Este problema ha sido también abordado por Ausubel, Novak y Hanesian (2011). El problema medular no es la edad sino la estimulación que reciben los niños y el comprender que cada uno tiene ritmos evolutivos distintos; en consecuencia, lo que se tendría que hacer es evaluar a cada uno y ubicarlo en el lugar que corresponda para su mejor desarrollo.

Así parece claro que un programa que estimula la psicomotricidad y de manera especial la coordinación visomanual, la orientación espacial y la estructura espacio temporal, que son funciones perceptivo motoras, influiría de modo positivo en la madurez para la lectoescritura con lo cual el estudiante de inicial que pasa a primaria, tendría mayores posibilidades de éxito.

Por otro lado, el hecho de que se hayan encontrado diferencias tanto en el grupo experimental como en el control entre las evaluaciones pre y posexperimentales en la prueba de autocontrol, probaría que los estímulos a los que están sometidos los niños en las aulas del nivel inicial permiten desarrollarlo y que posiblemente a ese respecto no es imprescindible un programa como el que se ha experimentado aquí, aun cuando los miembros del grupo experimental 
han revelado mayor cambio que los que mostraron los miembros del grupo control. Esto significa que las actividades que se desarrollan en el nivel inicial, sí estarían logrando un efecto positivo en el hecho de que el niño aprenda a controlar sus impulsos; lo cual en términos neurológicos implica mayor madurez y en términos conductuales mayor autorregulación de la conducta. Esto habla a favor, por lo menos en estos aspectos, de la estimulación que vienen recibiendo los estudiantes en el nivel.

En efecto, en las aulas del nivel inicial, los niños encuentran condiciones distintas a las de su hogar, y deben adaptarse a ellas asimilando nuevas normas de conducta. Ahora bien, el someterse a una regla exterior, supone un control del impulso espontáneo y constituye el requisito para la autorregulación de la conducta. Neurológicamente esto no es imposible, ya que el cerebro del pequeño de 4 años tiene una notable madurez anatómica y funcional que va a ser el sustento material de los logros que tendrá este niño (Lubliankaia, 1971). Se inicia el proceso en el que las principales y más elevadas formas del comportamiento humano y en particular de la actividad psíquica, van a tener como sustento neuroanatómico a los lóbulos frontales, tal es la frontalización de las funciones. Es en estas estructuras que se van a apoyar funciones tan importantes como son: crear planes y programas de acción, regular la actividad y verificar los resultados de las mismas (Luria, 1974), funciones que irán apareciendo gradualmente en el niño.

En el contexto del aula, el niño aprende a aceptar las normas, lo cual favorece el control de sus impulsos emocionales. Pero, si no lo aprendiera ya sea porque no hay normas o porque las hay muy pocas, es poco probable que desarrolle lo que Piaget (1974) ha denominado respeto unilateral basado en la regla coercitiva. He ahí la importancia de la inserción temprana del niño a la educación formal ya que en ella se generan las condiciones para el posterior paso a la etapa del respeto mutuo, basado en la regla racional.

Probablemente pueda ayudar a explicar el fenómeno, lo reportado por Rezabala (2012) en el sentido de que la actividad lúdica tiene una enorme influencia en el desarrollo socioafectivo de los niños. A este respecto hay que recordar que tanto en las aulas del nivel inicial como del programa se desarrollan actividades lúdicas. Y sería pertinente impulsar un trabajo como el de Amaya (2014) para que también los padres comprendan la importancia de la actividad lúdica en el desarrollo socioemocional de sus hijos.

En lo concerniente a la aceptación por los pares, en este trabajo, se entiende que estas se expresan en tanto y en cuanto el niño es aceptado y preferido por los demás; por ello se empleó el sociograma. En el grupo control, no son aceptados el 57 \% en la evaluación de entrada y en la evaluación de salida disminuye al 50 $\%$. Los aceptados y muy aceptados suman solo el 6,52 $\%$ en la entrada y en la salida $8,7 \%$. En realidad, el mayor desplazamiento se produjo desde no aceptado a poco aceptado que en la evaluación de entrada tuvo $34,39 \%$ y en la salida $41,3 \%$. Como puede apreciarse esos son los 7 puntos que disminuyeron en la salida del nivel no aceptado. Estos resultados llevados a ji al cuadrado de Pearson, no obstante, revelan diferencias significativas (tabla 11).

Ahora bien, el grupo experimental muestra diferencias más ostensibles. En efecto, en el nivel no aceptado en la evaluación preexperimental se encontraba el $79,7 \%$ de la muestra mientras que en la evaluación posexperimental este porcentaje cayó hasta el 26,3\% (casi a la tercera parte). Por otro lado entre aceptados y muy aceptados en la evaluación preexperimental solo se hallaba el 3,1\%; pero en la evaluación posexperimental se elevó hasta el 22,52 \%. La Ji cuadrado revela que estas diferencias son significativas (tabla 12). Esto implicaría que si bien las actividades propias de la educación inicial por si sola generan diferencias significativas en los índices de aceptación de los niños y por consiguiente en sus habilidades sociales.

El programa "estimulando el cerebro y movimiento del niño" tiene mucho mayor efecto positivo que aquellas, lo cual es comprensible si se recuerda que el programa contiene actividades lúdicas de carácter grupal que genera condiciones para que los pequeños interactúen más y se cohesionen para lograr los objetivos de la actividad. Pero además debe aceptar a otros niños y ser aceptado por ellos, esto requiere socializarse y por consiguiente desarrollar las habilidades que ello implica. Esto es muy importante porque como lo demostraron Lacunza, Castro y Contini (2009) el desarrollo de habilidades sociales incide en la autoestima, en la adopción de roles, en la autorregulación del comportamiento y el rendimiento académico, entre otros aspectos, aun en niños pobres. Nuestros hallazgos se condicen con los de Llanos (2006), Olivos (2010) y; Gutierrez y Villatoro (2011) y refuerzan la idea de que el desarrollo psicomotor es muy importante también en el desarrollo de habilidades sociales, tal como lo demostró Cotom en el 2012.

Las conclusiones son:

1. Al haberse establecido que no existen diferencias estadísticas entre las evaluaciones pre y posexperimentales del grupo control en psicomotricidad, pero sí las hay en el grupo experimental, se puede concluir que el programa "estimulando el cerebro y movimiento del niño" favorece el desarrollo de la psicomotricidad de los estudiantes del nivel inicial de Huancayo de tres, cuatro y cinco años. 
2. Al haberse establecido que tampoco hay diferencias entre las evaluaciones pre y posexperimentales del grupo control en la prueba de madurez para la lectoescritura, pero si las hay en el grupo experimental, se puede concluir que sí se desarrolla la psicomotricidad, este desarrollo influye positivamente en la madurez para la lectoescritura y que no basta la estimulación psicomotriz que vienen recibiendo en las aulas los pequeños, se requiere un programa especial que se aplique sistemáticamente, de lo contario no se puede asegurar que el niño que es promovido al primer grado tan solo por cumplir el requisito de la edad cronológica tenga la madurez que le permita enfrentar con éxito las exigencias del primer grado.

3. En lo referente al autocontrol, al haberse hallado diferencias estadísticas entre las evaluaciones pre y posexperimentales en ambos grupos, se puede concluir que los estímulos proporcionados por las actividades que se desarrollan en las aulas del nivel inicial, sí permiten que el pequeño aprenda a autorregular su conducta y aunque el programa optimiza esto, es prescindible para tales efectos en el nivel inicial.

4. Finalmente, el hecho de haber encontrado que en ambos grupos hay diferencias significativas entre las evaluaciones pre y posexperimentales en la aceptación, permite concluir que también aquí, es muy importante el papel que tiene la educación inicial pero que este es potencializado por el programa "estimulando el cerebro y movimiento del niño"; de modo que el uso del programa en el nivel inicial, por lo menos en la población estudiada, es recomendable.

\section{REFERENCIAS BIBLIOGRÁFICAS}

Alanya, C., y Núñez, E. (2006). Los videojuegos y el desarrollo de la coordinación visomanual en preescolares de 5 años que asisten a instituciones educativas estatales de la zona urbana de Huancayo (tesis de licenciatura). Universidad Nacional del Centro del Perú, Huancayo.

Alvino, R. (2010). Sumillado y comprensión lectora en estudiantes de sexto grado de instituciones educativas públicas de Huancayo (tesis). Universidad Nacional del Centro del Perú, Huancayo.

Amaya, L. (2014). Desarrollo de la afectividad en los niños del grado preescolar del gimnasio Ismael Perdomo (tesis). Universidad de Tolima. Recuperado de http://repository.ut.edu.co/ bitstream/001/1443/1/RIUT-JCDA-spa-2015 Desarrollo\%20de\%20la\%20afectividad\%20en\%20
los\%20ni\%C3\%B1 os\%20 del\%20grado\%20 preescolar $\% 20$ del\%20Gimnasio\%20lsmael $\% 20$ Perdomo.pdf

Arana, S. (2014). Autocontrol y su relación con la autoestima en adolescentes (tesis de grado) Universidad Rafael Landívar. Recuperado de http:// biblio3.url.edu.gt/Tesario/2014/05/42/AranaSharon.pdf

Arias, W., y Caycho, T. (2013). Análisis psicométrico de la prueba de diagnóstico de la madurez para el aprendizaje de la lectoescritura de Filho. Revista Psicología, 3(3), 25-47. Recuperado de http:// ucsp.edu.pe/investigacion/psicologia/wpcontent/ uploads/2014/01/- RevistadePsicolog\%C3\%ADa2do-art\%C3\%ADculo.pdf

Ausubel, D., Novak, J., y Hanesian, H. (201 1). Psicología Educativa. Un punto de vista cognoscitivo. México: Trillas.

Blas M., Javier, L., y Yarlequé, L. (1989). El desarrollo psicomotor de los niños de 2 a 5 años que viven en el penal Santa Mónica. Lima: CISEPS.

Celada, J., y Cairo, E. (1990). Actividad psíquica y cerebro. Lima: Concytec.

Condemarín, M. (1984). Madurez escolar. Santiago de Chile: Andrés Bello.

Contratarán a más de 4.500 profesores de educación física para dos mil escuelas (20 de enero de 2015). La República. Recuperado de http://larepublica. pe/21-01-2015/contrataran-a-mas-de-4500profesores-de-educacion-fisica-para-2-milescuelas

Cotom, L. (2012). Psicomotricidad y su relación en el proceso de lectoescritura. Recuperado de http:// biblio3.url.edu.gt/Tesario/2012/05/09/CotomLoreny.pdf

Chávez, K., y Ramos, D. (2014). Influencia familiar en el desarrollo de las competencias para iniciar el primer grado de primaria, en los infantes de cinco años de cuatro instituciones educativas del distrito de Florencia de Mora, Trujillo, año 2013 (tesis). Universidad Privada Antenor Orrego, Trujillo. Recuperado de http://repositorio.upao.edu. pe/bitstream/upaorep/901/1/CHAVEZ_KEILY INFLUENCIA_FAMILIAR_COMPETENCIAS.p̄jf

De la Cruz, L. (1989). Relación entre la percepción visomotriz y la madurez para el aprendizaje escolar en grupo de niños de 5 a 6 años de educación inicial del CEI 091-USE 13 (tesis). Universidad de San Martin de Porres, Lima.

Fernandes, F., Marín, F., y Urquijo, S. (2010). Relación entre los constructos autocontrol y autoconcepto en niños y jóvenes. Recuperado de http://www.scielo. org.pe/pdf/liber/v16n2/a11v16n2.pdf

Filho, L. (1925). Test ABC de verificación de la madurez necesaria para el aprendizaje de la lectura y escritura. Buenos Aires: Kapelusz.

Frostig, M. (1980). Programa para el desarrollo de la percepción visual. Buenos Aires: Panamericana.

Gastiaburú, G. (2012). Programa "Juego, coopero y 
aprendo" para el desarrollo psicomotor de niños de 3 años de una institución educativa, Callao (tesis magistral). Universidad San Ignacio de Loyola, Lima. Recuperado de http://repositorio. usil.edu.pe/wp-content/uploads/2014/07/2012 Gastiabur\%C3\%BA_Programa-Juego-cooperoy-aprendo-para-el-desarrollo-psicomotor-deni\%C3\%B los-de-3-a\%C3\%B 1 os-de-una-IE-delCallao.pdf

Gómez, I., y Luciano, M. (1991). Autocontrol en niños: Un estudio experimental sobre dos procedimientos en la adquisición de conductas de espera. Psicothema, 1(3), 25-44. Recuperado de http:// www.psicothema.com/pdf/2002.pdf

González, M., y Romero, J. (1999). ėlnfluye el desarrollo de la producción fonológica en el aprendizaje de la lectura? Rev Logop Fon Audiol 1999, XIX(2), 61-68. Recuperado de file://C:/Users/WIN/Downloads/ S0214460399757050 S300_es\%20(1).pdf

Guevara, B. (1980). Estudio experimental de un programa de aprestamiento especial en dos grupos de niños de cinco años con madurez inferior para el aprendizaje de la lectoescritura (tesis de licenciatura). Universidad Femenina del Sagrado Corazón, Lima.

Gutiérrez, M., y Villatoro, J. (2011). Desarrollo de habilidades sociales en la escuela. Revista Iberoamericana, 16(30), 34-40. Recuperado de http://www.uade.inpsiquiatria.edu.mx / Articulos\%20Jorge/2011/Desarrollo\%20de\%20 habilidades $\% 20$ sociales $\% 20$ en $\% 201$ a $\% 20$ escuela. pdf

Herrera, L., y Tejidor, S. (2004). Desarrollo del autocontrol en niños con comportamiento asociado al TDA/H (tesis). Universidad Católica Andrés Bello, Caracas. Recuperado de http:// biblioteca2.ucab.edu.ve/anexos/biblioteca/marc/ texto/AAQ0556\%20.pdf

Javier, L. (1988). Efectividad de un programa de juegos en el desarrollo de la atención en niños con problemas de aprendizaje (tesis). Universidad Ricardo Palma, Lima.

-------- (1994). Estudio del desarrollo psicomotor de niños en edad preescolar de las ciudades de Huancayo (departamento de Junín) y Bellavista (departamento de San Martin) (tesis). Universidad Ricardo Palma, Lima.

Kazdin, E. (2001). Métodos de investigación en psicología clínica (3a ed.). México: Pearson Educación.

Lacunza, A. B., Castro, A., y Contini, N. (2009). Habilidades sociales preescolares: una escala para niños de contextos de pobreza. Revista de Psicología, XXVII (1), 4-28. Recuperado de http:// www.redalyc.org/articulo.oa? id $=337829512001$

Llanos, C. (2006). Efectos de un programa de enseñanza en habilidades sociales (tesis). Universidad de Granada, España. Recuperado de http://hera.ugr.es/tesisugr/15885574.pdf.
Liublinskaia, A. (1971). Desarrollo psíquico del niño preescolar. México: Editorial Grijalbo

Luria, A. (1974). El cerebro en acción. Barcelona: Fontanella.

Maco, E. (2015). Plan Leo y comprensión lectora en estudiantes de primaria de Chincha (tesis magistral). Universidad Nacional del Centro del Perú, Huancayo.

MINEDU (2016). Oficina de Medición de la Calidad de los Aprendizajes. Evaluación Censal de Estudiantes 2015. Recuperado de http://umc.minedu.gob.pe/ evaluacion-censal-de-estudiantes-ece-2015/

MINEDU (2014). Resolución Ministerial N556-2014MINEDU Recuperado de http://www. minedu.gob. pe/campanias/pdf/Resolucion_Ministerial_5562014-MINEDU.pdf

Nieto, M. (1980). El niño disléxico. México: Prensa Médica

Núñez, E. R. (2015). Evidencias empíricas de la teoría vigotskiana acerca del aprendizaje y desarrollo (tesis doctoral). Universidad Nacional del Centro del Perú, Huancayo.

PISA (2015). PISA 2015. Resultados clave. Recuperado de https://www.oecd.org/pisa/pisa-2015-resultsin-focus-ESP.pdf

Olivos, V. (2010). Entrenamiento de habilidades sociales para la integración psicosocial de 4

Oramas, L. (2000). Propuesta de un programa de práctica psicomotriz para niños de 2 a 3 años (tesis de licenciatura). Facultad de Ciencias y Artes Escuela de Educación, Universidad Metropolitana, Caracas. Recuperado de http://repositorios. unimet.edu.ve/docs/ 34/LB1 140073P4.pdf

Pacheco, P., y Romero, K. (2005). Programa pastos para la estimulación psicomotora gruesa en niños de tres años de la zona urbana de Huancayo (tesis de licenciatura). Universidad Nacional del Centro del Perú, Huancayo.

Papalia, D., Wendkos, S., y Duskin, R. (2010). Desarrollo humano (11 ed.). México: McGraw-Hill Educación.

Pérez, R. (2004). Psicomotricidad, desarrollo psicomotor en la infancia. España: Ideas Propias Editorial. Recuperado de http://media.axon.es/ pdf/90072.pdf

Piacente, T., Marder, S., Resches, M., y Ledesma, R. (2005). El contexto alfabetizador hogareño en familias de la pobreza. Comparación de sus características con las de familias no pobres. RIDEP, 1(21), 61-88. Recuperado de http://www.aidep. org/03_ridep/R21/R214.pdf

Piaget, J. (1974). El criterio moral en el niño. Barcelona: Fontanella.

Pugh, K. (julio, 2014). La relación entre lo fonológico y el procesamiento auditivo y la organización del cerebro en los lectores principiantes. Congreso de Neuroeducación, Lima, Perú

Rezabala, M. (2012). La recreación infantil y su incidencia en el desarrollo socio afectivo de los niños 
y niñas del centro de educación inicial "Luz y Vida" del recinto unidad nacional del Canton Putumayo, provincia de Sucumbios (tesis). Universidad Nacional de Loja, Ecuador, Recuperado de http://dspace. unl.edu.ec/jspui/bitstream/123456789/244/1/ TESIS\% 20MAR\%C3\%8DA\%20MARTIBEL\%20 REZABALA\%20PLAZA.pdf

Salazar, N. (2013). La psicomotricidad y su incidencia en la lectoescritura de los niños y niñas del primer año de educación básica del jardín de infantes "Club Árabe Ecuatoriano" de la ciudad de Quito, período lectivo 2012-2013 (tesis). Universidad Nacional de Loja, Quito. Recuperado de https://dspace.unl. edu.ec/ispui/bitstream/123456789/834/1/TESIS. pdf

Sánchez, H., y Reyes, C. (julio, 2015). Congreso Interamericano de Psicología. Lima-Perú

Sánchez, H., y Reyes, C. (2006). Metodología y diseños en la investigación científica ( $2 a$ ed.). Lima: Visión Universitaria.

Serrano, M., y García, D. (2010). Inteligencia emocional: autocontrol en adolescentes estudiantes del último año de secundaria. Revista Multiciencias, 10(3), 273-280. Recuperado de http://www.redalyc.org/ pdf/904/90416328008.pdf

Soto, N. (1986). Estudio del desarrollo de la percepción visomotriz y su relación con la madurez en niños de edad preescolar de diferentes niveles socioeconómicos (tesis). Universidad Ricardo Palma, Lima.
Suárez, M. (1999). Madurez escolar para la lectoescritura mediante el test $A B C$ y factores relacionados en niños preescolares de dos instituciones privadas y dos públicas (tesis). Universidad Centrooccidental Lisandro Alvarado (UCLA), Venezuela. Recuperado de http://bibmed. ucla.edu.ve/edocs_bmucla/textocompleto/tw4 dv4s837m.pdf

Yarlequé, L., y Cerrón, A. (2011). Investigación educativa. Huancayo: Programa de Complementación Académica y Licenciatura de la Universidad Nacional del Centro del Perú.

Yarlequé, L. (1998). Psicología Evolutiva y Pedagógica. Huancayo: Universidad Nacional del Centro del Perú.

Zavala, M. (1991). Desnutrición y desarrollo psicomotor en niños en edad preescolar de la zona urbano marginal de Lima Metropolitana. (tesis). Universidad Ricardo Palma, Lima.

Zavala, M., Valadez, M., y Vargas, M. (2008). Inteligencia emocional y habilidades sociales en adolescentes con alta aceptación social. Electronic Journal of Research in Educational Psychology, 6(2), 321-338. Recuperado de http://www.redalyc.org/ articulo.oa? id $=293121924004$ 